\title{
Diffraction of coherent phonons emitted by a grating
}

\author{
D. J. Dieleman, A. F. Koenderink, A. F. M. Arts, and H. W. de Wijn \\ Faculty of Physics and Astronomy, and Debye Research Institute, Utrecht University, P.O. Box 80.000, \\ 3508 TA Utrecht, The Netherlands
}

(Received 20 May 1999)

\begin{abstract}
Coherent acoustic phonons emitted by a grating are observed in the gigahertz range, and found to show well-resolved interference maxima on either side of the forward direction. The phonons are generated by laser-induced periodic heating of a thin Au transducer, consisting of an array of narrow strips deposited onto a (001) face of a single crystal of $\mathrm{PbMoO}_{4}$. The phase and group velocities, which are noncollinear due to the elastic anisotropy, are distinguished with reliance on Brillouin scattering. The results are in accord with optical diffraction theory in the Fraunhofer limit, provided phonon defocusing is taken into account.
\end{abstract}

[S0163-1829(99)01541-6]

\section{INTRODUCTION}

Diffraction by gratings, starting with Young's original two-pinhole arrangement, is one of the decisive experiments in establishing the wave character of light. The incident wave front is split over an array of parallel slits, each slit acting as a new light source. ${ }^{1}$ The resulting intensity distribution measured at some distance shows crests and troughs due to interference, provided the light is sufficiently monochromatic and sufficiently spatially coherent. The present paper is intended to demonstrate that similar experiments can be conducted with acoustic waves in the gigahertz range, thus advancing the relatively new field of "phonon optics." Monochromatic acoustic waves coherently emitted by an array of strip-shaped sources are shown to engage in constructive and destructive interference. As distinct from standard optics, however, the phase and group velocities are noncollinear as a result of the elastic anisotropy of the crystalline material. The experiment is therefore set up such that the direction in which the energy is transported can be distinguished from the acoustic wave vector.

The present experiment has become feasible after the development of the technique of generation of coherent acoustic waves by cw laser-induced thermomodulation of a thin metallic transducer evaporated onto the sample. ${ }^{2}$ Two singlefrequency $\mathrm{cw}$ dye lasers, operating at a small difference frequency in the gigahertz range, are collinearly focused onto the transducer, to produce a periodic heating of its surface. The resulting periodic thermal expansion generates a coherent strain wave at the difference frequency, which is ultimately injected into the crystal as a longitudinally polarized acoustic wave. To form a grating, the transducer consists of parallel metallic strips of a width comparable to the acoustic wavelength rather than a uniform metallic film. The strips, serving as the slits of the grating, are coherently excited by the optical heating. As for the detection, use is made of Brillouin scattering because of its selectivity for the wave vector. A single crystal of lead molybdate $\left(\mathrm{PbMoO}_{4}\right)$ was chosen as sample for reasons of its transparency and high Brillouin efficiency.

\section{EXPERIMENTS}

The two interfering dye-laser beams, injecting longitudinal acoustic phonons into the crystal at their difference frequency, each delivered typically $400 \mathrm{~mW}$ of light in the red $(\lambda \approx 590 \mathrm{~nm})$. Their beams were jointly focused onto the grating-shaped transducer by a single 80 -mm lens, to form a temperature-modulated spot approximately $20 \mu \mathrm{m}$ in radius. As the generated strain scales with the optical intensity squared, the resultant acoustic beam is slightly more narrow, reaching a minimum $1 / e$ radius of $15 \mu \mathrm{m}$. Somewhat larger radii were obtained by shifting the focusing lens along the optical axis.

The specimen is a cuboid-shaped single crystal of $\mathrm{PbMoO}_{4}$, approximately $10 \times 10 \times 5 \mathrm{~mm}^{3}$ in size. The $c$ axis is parallel to the larger sides. To fabricate a transducer in the form of a grating, first a uniform 600-nm thick Au film was deposited onto a crystal face perpendicular to the $c$ axis, with a thin $(5 \mathrm{~nm})$ intermediate layer of $\mathrm{Cr}$ for better adhesion. Subsequent photoetching left a periodic array of Au strips $3.2 \pm 0.1 \mu \mathrm{m}$ in width with a grating period of $8.00 \pm 0.02$ $\mu \mathrm{m}$. The grating period was verified by diffraction of light from a He-Ne laser. The sample was held at room temperature.

The acoustic waves injected into the crystal were detected with Brillouin scattering. The primary Brillouin laser beam was provided by an argon-ion laser operating in the singleline mode, and delivering $200 \mathrm{~mW}$ of light at a wavelength of $514.5 \mathrm{~nm}$. It was focused down to a waist approximately $15 \mu \mathrm{m}$ in diameter, which, along with the optics receiving the scattered light, delimits the detection volume to a 400 $\mu \mathrm{m}$-long pencil. The scattered light was frequency analyzed by the use of a quintuple-pass Fabry-Perot interferometer, followed by a monochromator to remove residual Raman and laser-plasma lines, a cooled photomultiplier, and standard photon-counting and data-accumulation techniques. The interferometer was actively stabilized to the frequency of the longitudinal acoustic anti-Stokes peak. The angular resolution of the detected acoustic wave vector is determined by the divergence of the primary Brillouin beam, the finite size of the detection volume, and the divergence of the detected scattered light. In the present arrangement, it amounts to 
(a)

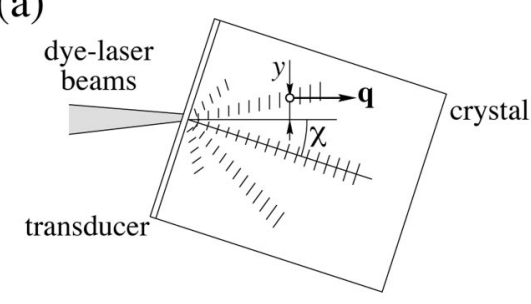

(b)

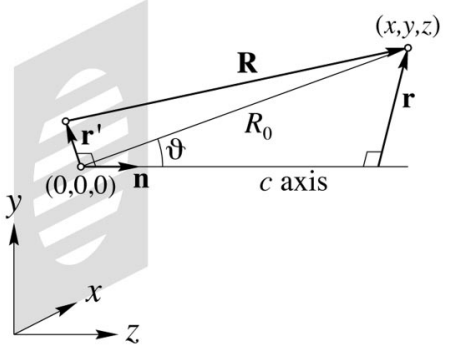

FIG. 1. (a) The geometry as seen from the side. The interfering dye-laser beams are focused on a microscopic Au grating acting as transducer. To achieve Brillouin scattering from a noncentral diffraction maximum, the crystal is rotated over an angle $\chi$ such that $\mathbf{q}$ and $\mathbf{v}_{\text {phase }}$ lie in the Brillouin scattering plane. The illuminated spot at the transducer is subsequently shifted downward over a distance $y$ until $\mathbf{v}_{\text {group }}$ passes through the Brillouin detection volume (open circle). (b) The grating, represented by white stripes, is limited to a circular area by the Gaussian intensity distribution of the dye lasers. The coordinates refer to Sec. III.

about $\pm 0.5^{\circ}$, i.e., less than $\pm 2 \mu \mathrm{m}$ at the distance of about $200 \mu \mathrm{m}$.

The scattering geometry is depicted in Fig. 1(a). The grating strips are horizontal, and so is the scattering plane defined by the incoming and outgoing Brillouin light. For noncentral diffraction maxima, the phonon wave vector $\mathbf{q}$ points away from the $c$ axis. Furthermore, the group velocity differs in direction from the phase velocity beyond the angular resolution. It is necessary therefore to review the conditions for optimal Brillouin signals in some detail. The conditions to be fulfilled are: (i) the acoustic wave vector, which closes the incoming and outgoing optical wave vectors, must lie in the scattering plane, and (ii) the acoustic intensity, which propagates with the group velocity, must pass through the Brillouin detection volume. Repositioning of the Brillouin scattering plane and the detection volume is quite cumbersome, so that it is best to leave the Brillouin setup fixed. To achieve (i), therefore, the crystal was mounted on a rotation stage, which permitted to tilt the crystal about an axis parallel to the grating strips and to reorient the wave vector into the scattering plane. The group velocity, however, may point in a direction closer to the $c$ axis (phonon focusing), or, as in the present case, further away from it (defocusing). Additional upward or downward displacement of the acoustic beam is necessary to ensure its passage through the Brillouin detection volume, and to meet condition (ii). This was achieved by vertical displacement of the joint dye-laser focus until maximum signal was reached. In practice, the locus of the focal spot was controlled by lateral displacement of the focusing lens. Note that the horizontal position is less critical because the detection volume is pencil shaped. The proce-

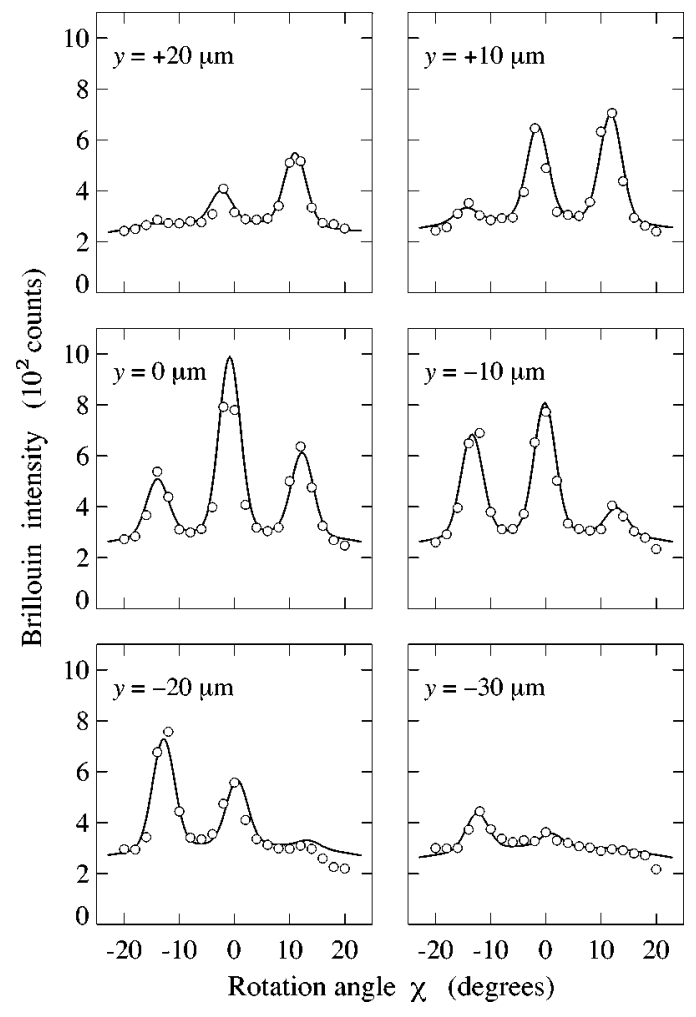

FIG. 2. Brillouin intensity profiles as a function of the rotation angle $\chi$ for various displacements $y$. The frequency of the acoustic wave is $\omega / 2 \pi=2.2 \mathrm{GHz}$, and the detection volume is at a depth $z$ $=188 \mu \mathrm{m}$ below the transducer. The grating is irradiated with a circular Gaussian intensity profile, with $1 / e$ width $w=21 \pm 2 \mu \mathrm{m}$. The solid curves are fits based on Eq. (7) convoluted with the resolution.

dure outlined permits us to retrieve the directions of both the phase and group velocities.

When measuring the diffraction profiles, the relevant degrees of freedom therefore are the rotation angle $\chi$ of the crystalline $c$ axis relative to the Brillouin scattering plane and the height $y$ of the Brillouin plane above or below the joint dye-laser focus. We have chosen to scan $\chi$ for a series of fixed values of $y$ centered about zero. Prior to these scans, the position of the joint dye-laser focus was optimized by maximizing the Brillouin intensity associated with the central interference maximum. The angle $\chi$ was typically advanced from $-20^{\circ}$ to $+20^{\circ}$ in steps of $2^{\circ}$, while $y$ ranged from -30 to $+30 \mu \mathrm{m}$. The interference maxima were recorded at distances $z=73,188$, and $388 \mu \mathrm{m}$ below the transducer, where, again to leave the Brillouin setup fixed, $z$ was changed by displacing the complete assembly of crystal, rotation stage, and focusing lens. A representative set of data measured at a phonon frequency of $2.2 \mathrm{GHz}$ is shown in Fig. 2.

The actual geometry is slightly more complex than depicted in Fig. 1(a) in that the rotation axis lies at a short distance $z_{0}$ in front of the transducer. The angle $\vartheta$ at which the detection volume is seen from the center of the grating then reads, with reference to the $c$ axis,

$$
\vartheta=\chi+\arcsin \left[\left(y+z_{0} \sin \chi\right) / z\right],
$$

which for small angles reduces to the more transparent relation $\vartheta=\left[\left(z+z_{0}\right) \chi+y\right] / z$. A positive $z_{0}$ reduces the $y$ neces- 
sary to achieve the optimum $\vartheta$, and therefore helps to observe the central and first-order diffraction maxima in a single $\chi$ scan. The value for $z_{0}$ can be extracted by the use of Eq. (1) from the $z$ dependences of $\chi$ and $y$ associated with a fixed well-defined $\vartheta$, such as the $\vartheta$ associated with a firstorder maximum. The result is $z_{0}=40 \pm 10 \mu \mathrm{m}$.

\section{FRAUNHOFER DIFFRACTION AND PHONON FOCUSING}

In this section, the interference pattern resulting from diffraction by multiple slits is calculated in the Fraunhofer approximation with due account of the effects of phonon focusing. The starting point is the generalized Kirchhoff integral for Dirichlet boundary conditions, ${ }^{3,4}$ which enables us to evaluate the strain $\varepsilon(x, y, z)$ at any position $(x, y, z)$ in the crystal from a given distribution of the strain $\varepsilon\left(x^{\prime}, y^{\prime}, 0\right)$ in the plane of the transducer $(z=0)$. Dropping the harmonic time dependence $e^{i \omega(\mathbf{q}) t}$, we have

$$
\begin{aligned}
\varepsilon(x, y, z)= & \frac{1}{2 \pi i} \int_{-\infty}^{+\infty} \int_{-\infty}^{+\infty} \varepsilon\left(x^{\prime}, y^{\prime}, 0\right) \\
& \times \frac{e^{-i q R}}{R} q\left(1+\frac{i}{q R}\right) \frac{\mathbf{n} \cdot \mathbf{R}}{R} d x^{\prime} d y^{\prime} .
\end{aligned}
$$

As inside the crystal $\varepsilon(x, y, z)$ satisfies a homogeneous wave equation, it is assumed that Eq. (2) applies to each component of the strain tensor. The coordinate system adopted is shown in relation to the geometry of the grating in Fig. 1(b). The vector $\mathbf{R}$ connects the surface element $d x^{\prime} d y^{\prime}$ of the source with the point of observation $(x, y, z)$, while $\mathbf{n}$ is the normal unit vector. The origin is, for convenience, chosen midway between two Au strips. This choice is inconsequential because the period of the grating is small in comparison with its size.

Phonon focusing, or defocusing, results from the fact that the slowness surfaces, i.e., surfaces in $\mathbf{q}$ space of constant dispersion $\omega(\mathbf{q})$, are anisotropic. ${ }^{5-7}$ In the present geometry, the central direction of propagation, the $z$ axis, coincides with the fourfold crystalline $c$ axis. The variation of the modulus of $\mathbf{q}$ when moving along a slowness surface may then be accommodated into a single parameter $p$ through $^{8}$

$$
q=q_{c}\left(1-p \vartheta_{\mathbf{q}}^{2}\right),
$$

where $\vartheta_{\mathbf{q}}$ is the angle of $\mathbf{q}$ from the $c$ axis, and $q_{c}$ is the modulus of $\mathbf{q}$ along this axis; $\vartheta_{\mathbf{q}}=\arctan \left(\left|\mathbf{r}-\mathbf{r}^{\prime}\right| / z\right)$, where $\mathbf{r}=(x, y)$ and $\mathbf{r}^{\prime}=\left(x^{\prime}, y^{\prime}\right)$ are two-dimensional vectors lying in the "screen" of observation and the transducer, respectively [cf. Fig. 1(b)]. For a given surface of constant dispersion $\omega(\mathbf{q})=\omega$, we have $q_{c}=\omega / \mathrm{V}_{c}$, with $\mathrm{V}_{c}$ the velocity of longitudinal sound along the $c$ axis. In $\mathrm{PbMoO}_{4}, \mathrm{v}_{c}=3.63$ $\mathrm{km} / \mathrm{s}$. The focusing parameter $p$ may be related to the elastic constants $C_{i j}$ through an analysis of the Christoffel equations. ${ }^{8}$ For longitudinal acoustic phonons propagating in directions near a fourfold $c$ axis,

$$
p=\frac{C_{13}^{2}+2 C_{13} C_{44}+2 C_{33} C_{44}-C_{33}^{2}}{2 C_{33}\left(C_{33}-C_{44}\right)} .
$$

From known values for $C_{i j},{ }^{9}$ this yields $p=+0.174$, i.e., defocusing in $\mathrm{PbMoO}_{4}$. Equation (3) is adequate up to angles $\vartheta_{\mathrm{q}} \approx 25^{\circ}$.

The experiments were conducted in the Fraunhofer regime, far away from the grating in measure of the acoustic wavelength $(\lambda \approx 1.7 \mu \mathrm{m})$ and the grating dimensions, and up to moderate angles from the $z$ axis $\left(\lessgtr 20^{\circ}\right)$. At this point, therefore, we substitute Eq. (3) into Eq. (2), expand the exponent in $e^{-i q R}$ up to first order in $x^{\prime}$ and $y^{\prime}$, take the zerothorder term, which contains the phase with reference to the origin, out of the integral, and subsequently expand the firstorder term up to first-order in $x$ and $y$. The remainder of the integrand is to sufficient accuracy approximated by $\varepsilon\left(x^{\prime}, y^{\prime}, 0\right) q_{c} / z$. Noting that the point of observation $(x, y, z)$ is at an angle $\vartheta=\arctan (r / z)$ and a distance $R_{0}=\left(z^{2}+r^{2}\right)^{1 / 2}$ from the origin, we then find for the diffracted strain in the Fraunhofer approximation

$$
\begin{aligned}
\varepsilon(x, y, z)= & \frac{q_{c}}{2 \pi i z} e^{-i q_{c}\left(1-p \vartheta^{2}\right) R_{0}} \int_{-\infty}^{+\infty} \int_{-\infty}^{+\infty} \varepsilon\left(x^{\prime}, y^{\prime}, 0\right) \\
& \times e^{i q_{c}(1-2 p)\left(x x^{\prime}+y y^{\prime}\right) / z} d x^{\prime} d y^{\prime} .
\end{aligned}
$$

The strain source $\varepsilon\left(x^{\prime}, y^{\prime}, 0\right)$ is determined by the grating geometry combined with the optical intensity and the phase profiles of the interfering dye-laser beams. The optical intensity profile is known to be essentially Gaussian. Previous experiments with homogeneous transducers ${ }^{2}$ have established that the intensity distribution and the coherence of the light modulation are passed onto the acoustic beam. Thermomodulation only occurs in the strips. Upon assuming a uniform phase, the source term in Eq. (5) may thus be written

$$
\varepsilon\left(x^{\prime}, y^{\prime}, 0\right)=\varepsilon_{0} e^{-\left(x^{\prime 2}+y^{\prime 2}\right) / w^{2}} \Theta\left(y^{\prime}\right),
$$

where $\varepsilon_{0}$ is an overall amplitude, and the periodic block function $\Theta\left(y^{\prime}\right)$ delimits the strips. We have $\Theta\left(y^{\prime}\right)=1$ if $\left(n-\frac{1}{2}\right) d-\frac{1}{2} s \leqslant y^{\prime} \leqslant\left(n-\frac{1}{2}\right) d+\frac{1}{2} s \quad$ with $n$ integer, and $\Theta\left(y^{\prime}\right)=0$ elsewhere; $d$ is the grating parameter; $s$ is the strip width. The parameter $w$, which in the present experiments amounts to about $20 \mu \mathrm{m}$, measures the spatial extent of the strain wave near the transducer.

The integral over $y^{\prime}$ in Eq. (5) is amenable to rigorous evaluation if $\varepsilon\left(x^{\prime}, y^{\prime}, 0\right)$ is independent of $y^{\prime}$ over the extent of each individual strip. Without appreciable loss of accuracy since $s \ll w$, we therefore adopt for strip $n$ the midvalue $I_{n}$ $=\varepsilon\left(x^{\prime},\left(n-\frac{1}{2}\right) d, 0\right)$. Straightforward integration then yields

$$
\begin{aligned}
\varepsilon(x, y, z)= & \varepsilon_{0} \frac{q_{c} w s}{i \pi^{1 / 2} z} e^{-\left[q_{c}(1-2 p) w x / 2 z\right]^{2}} \\
& \times e^{-i q_{c}\left(1-p \vartheta^{2}\right) R_{0}} \frac{\sin \beta}{\beta} \sum_{n=1}^{\infty} I_{n} \cos \alpha_{n},
\end{aligned}
$$

where the geometry is contained in the angles

$$
\begin{gathered}
\alpha_{n}=q_{c}(1-2 p)\left(n-\frac{1}{2}\right) d \sin \vartheta, \\
\beta=\frac{1}{2} q_{c}(1-2 p) s \sin \vartheta .
\end{gathered}
$$

The summation runs over pairs of strips positioned symmetrically about the origin. Quite similarly to optical diffrac- 
tion by multiple slits, ${ }^{1}$ these pairs contribute with weights $I_{n} \cos \alpha_{n}$, while the factor $\sin \beta / \beta$ accounts for the diffraction by an individual strip. Because $w / d \approx 2.5$, the summation can be restricted to the first few terms. The first exponential in Eq. (7) confines the diffracted strain laterally with an angular $1 / e$ spread $\pm 2 / q_{c}(1-2 p) w \approx \pm 2.5^{\circ}$ about the vertical plane.

\section{RESULTS AND DISCUSSION}

The profiles presented in Fig. 2 show the anti-Stokes Brillouin intensity, i.e., the strain squared, of the longitudinal acoustic wave versus the tilt angle $\chi$ of the crystal for several vertical shifts $y$. As explained in Sec. II, $\chi$ is to be identified with the angle $\vartheta_{\mathbf{q}}$ the detected wave vector $\mathbf{q}$ makes with the $c$ axis. The data in Fig. 2 have been measured at a phonon frequency $\omega / 2 \pi=2.2 \mathrm{GHz}$, while the Brillouin detection volume is located at a depth of $z=188 \mu \mathrm{m}$. The intensitymodulated optical spot had a $1 / e$ radius $w=21 \pm 2 \mu \mathrm{m}$. This radius, effected by slightly displacing the dye laser focus away from the grating, is sufficient to cover, say, six strips. Each data point required only $10 \mathrm{~s}$ of accumulation time, the noise residing mainly in short-term variations of the laser intensities and the beam positions.

The salient result of Fig. 2 is that first-order maxima are observed on either side of the zeroth-order maximum. This demonstrates a substantial degree of coherence of the 2.2$\mathrm{GHz}$ acoustic wave. In fact, its spectral purity $(\leqslant 1 \mathrm{MHz})$ would be sufficient for coherence to extend over several millimeters, where it not that at room temperature the mean free path is shortened by anharmonic decay. ${ }^{10,11}$ The first-order maxima occur at $\vartheta_{\mathbf{q}} \approx \pm 12^{\circ}$. Contrary to standard optics, however, their intensities become unbalanced upon introducing a lateral displacement $y$. Already for $y$ as small as \pm 10 $\mu \mathrm{m}$, corresponding to $\pm 3^{\circ}$ in $\vartheta$, the $\chi$ scans in Fig. 2 bypass one of the first-order maxima. It is noted that a similar set of data, not reproduced here, for which the grating is diminished in size by a more precise focusing of the laser beams $\left(w_{0}=15 \pm 2 \mu \mathrm{m}\right)$, exhibits the same features, except that, as expected for a smaller grating, the diffraction peaks are broader by approximately $20 \%$ [cf. Eq. (7)]. In this context, it is furthermore noted that second and third-order diffraction maxima have been observed in a less complete set of data taken at $4.4 \mathrm{GHz}$.

The wave vectors associated with the first-order maxima, and the corresponding phase velocities $\mathbf{v}_{\text {phase }}$, appear to point in directions that are in agreement with standard diffraction theory, which predicts them to be at angles $\vartheta_{\mathbf{q}}$ given by

$$
q d \sin \vartheta_{\mathbf{q}}=2 \pi
$$

i.e., the paths departing from successive strips differ by $\lambda$. Note that this elementary interference condition is not modified by the elastic anisotropy, except for the directional dependence of $q=2 \pi / \lambda$. With Eq. (3) inserted, Eq. (10) yields $\vartheta_{\mathrm{q}}= \pm 12.0^{\circ}$, which equals the experimental value. The phase fronts are, however, not spherical, and as a consequence the maximum intensities, or the group velocities $\mathrm{v}_{\text {group }}$, are found in a different direction. To make this more tangible, we convert the data of Fig. 2 to a form that, in addition to the rotation angle $\chi$, explicitly shows the angle $\vartheta$ at which the maximum intensity is observed. Such a plot is

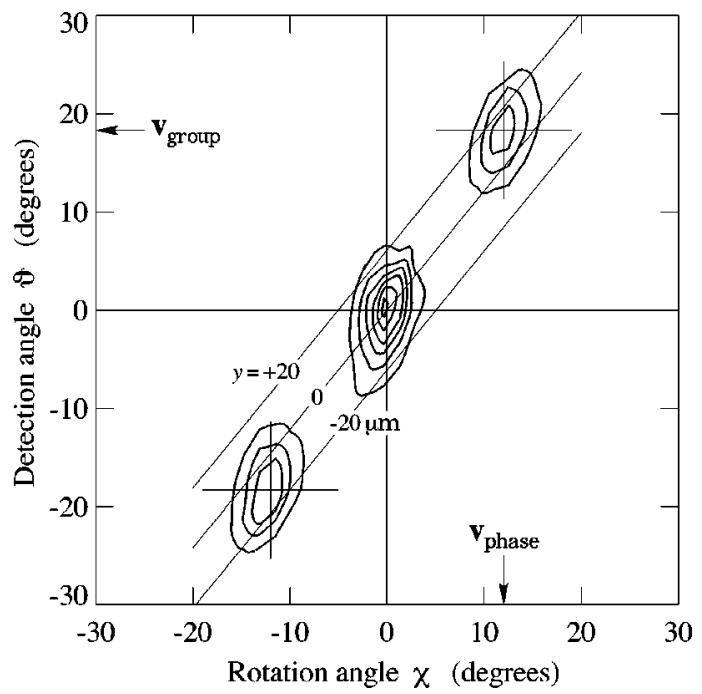

FIG. 3. Contour plot of the Brillouin intensity as a function of $\chi$ and $\vartheta$, i.e., the angles with which the experiment probes the phase and group velocities. The contours correspond to $17,33,50,67,83$, and $98 \%$ of the zeroth-order peak intensity. They are derived from the data in Fig. 2 after subtracting the background and changing over from $(\chi, y)$ to $(\chi, \vartheta)$ via Eq. (1). The first-order maxima occur at the cross points of $\vartheta_{\mathbf{q}}$ and $\vartheta_{g}$ predicted from Eqs. (10) and (11), marked by arrows. The straight lines correspond to the $y=-20,0$, and $+20-\mu \mathrm{m}$ scans in Fig. 2.

the contour diagram of the Brillouin intensity as a function of $\chi$ and $\vartheta$ presented in Fig. 3. The maximum first-order intensities are seen to occur at angles $\vartheta_{g} \approx \pm 18^{\circ}$ on either side from the central maximum. This result is to be compared with the first-order extrema of the acoustic intensity derived from Eq. (7). As the intensity directly scales with $|\varepsilon(x, y, z)|^{2}$, these maxima are expected at $\vartheta_{g}$ given by

$$
q_{c}(1-2 p) d \sin \vartheta_{g}=2 \pi,
$$

from which we find $\vartheta_{g}= \pm 18.4^{\circ}$ upon inserting the appropriate values for $q_{c}, d$, and $p$.

Figure 3 also shows to better advantage why the scans of the Brillouin intensity versus the rotation angle $\chi$ are asymmetric for $y \neq 0$. The three virtually linear trajectories inserted in Fig. 3, which are calculated with the aid of Eq. (1), correspond to the scans in Fig. 2 with $y=-20$, 0, and +20 $\mu \mathrm{m}$. The trajectories for $y= \pm 20 \mu \mathrm{m}$ are indeed seen to pass over the top of one first-order maximum, but to go wide of the maximum on the other side.

We conclude this section with a calculation of the profiles in Fig. 2, starting from Eq. (7). The Brillouin scattering intensity scales with $|\varepsilon(x, y, z)|^{2}$ integrated over the Brillouin detection volume with due account of the acceptance angle of the wave vector. The primary Brillouin beam has a Gaussian profile with a half $1 / e$ width of $7 \mu \mathrm{m}$, which at a depth of $188 \mu \mathrm{m}$ corresponds with a broadening of approximately $2^{\circ}$ in $\chi$ and $\vartheta$. The angular resolution for the wave vector is also assumed to be Gaussian, but adds to the angular broadening by only $0.5^{\circ}$. The finite resolution therefore accounts for about half of the linewidth in the scans of Fig. 2. The measured Brillouin intensity furthermore scales with the intensity of the primary Brillouin beam within the crystal, which varies with $\cos ^{2} \chi$ due to the optical anisotropy of 
$\mathrm{PbMoO}_{4}$. The curves plotted in Fig. 2 are fits of Eq. (7) convoluted with the finite resolution and augmented with the thermal background, which is assumed to scale with the intensity of the primary Brillouin beam. All parameters were taken from experiment, except for an overall vertical scaling factor. The calculated intensities generally trace the experimental data points. In particular, they reproduce the widths of the maxima and the asymmetry of the intensity for nonzero $y$.

\section{CONCLUSIONS}

In summary, we have demonstrated that coherent phonons emitted at gigahertz frequencies by a microscopic metallic grating show a diffraction pattern. The individual strips making up the grating served as coherent sources, much like the slits of an optical grating. As distinct from regular optics, however, the first-order interference maxima were observed at angles further out than the wave vector due to phonon defocusing associated with the elastic anisotropy. Fraunhofer diffraction theory with inclusion of the elastic anisotropy adequately accounts for the experimental findings.

\section{ACKNOWLEDGMENTS}

The authors thank M. de Bruin and N. Schneider of the ' 'Stichting Ruimte Onderzoek Nederland (SRON)', for etching the grating. The work was supported by the Netherlands Foundation "Fundamenteel Onderzoek der Materie (FOM)", and the "Nederlandse Organisatie voor Wetenschappelijk Onderzoek (NWO).,'
${ }^{1}$ M. Born and E. Wolf, Principles of Optics, 6th ed. (Pergamon Press, New York, 1980).

${ }^{2}$ E. P. N. Damen, A. F. M. Arts, and H. W. de Wijn, Phys. Rev. Lett. 74, 4249 (1995).

${ }^{3}$ P. M. Morse and H. Feshbach, Methods of Theoretical Physics (McGraw-Hill, New York, 1953), p. 812.

${ }^{4}$ J. D. Jackson, Classical Electrodynamics, 2nd ed. (Wiley \& Sons, New York, 1975), p. 428.

${ }^{5}$ B. Taylor, H. J. Maris, and C. Elbaum, Phys. Rev. B 3, 1462 (1971).

${ }^{6}$ J. P. Wolfe, Phys. Today 48 (9), 34 (1995).
${ }^{7}$ J. P. Wolfe, Imaging Phonons (Cambridge University Press, Cambridge, U.K., 1998).

${ }^{8}$ H. J. Maris, in Nonequilibrium Phonons in Nonmetallic Crystals, edited by W. Eisenmenger and A. A. Kaplyanskii (NorthHolland, Amsterdam, 1986), p. 51.

${ }^{9}$ G. A. Coquin, D. A. Pinnow, and A. M. Warner, J. Appl. Phys. 42, 2162 (1971).

${ }^{10}$ G. P. Srivastava, The Physics of Phonons (Adam Hilger, Bristol, 1990).

${ }^{11}$ E. P. N. Damen, A. F. M. Arts, and H. W. de Wijn, Phys. Rev. B 59, 349 (1999). 